\title{
Deformation-Induced Mechanical Anisotropy of Gelatin
}

\section{Films}

Weibing Teng, $†$ Xiangming Zhang, $†$ Valerie Merkle, $\neq$ Xiaoyi $W u^{*}, \dagger, \ddagger$

$\dagger$ Department of Aerospace and Mechanical Engineering, $\$$ Biomedical Engineering Interdisciplinary

Graduate Program and Bio5 Institute, The University of Arizona, Tucson, Arizona 85721, United States

of America

*To whom correspondence should be addressed. Tel.: 1-00-520-626-5854. Fax: 1-00-520-621-8198. Email: xwu@email.arizona.edu.

\section{RECEIVED DATE}




\section{ABSTRACT}

Deformation-induced mechanical and structural anisotropy has been analyzed using a gelatin film model. Specifically, gelatin films cast from water and 2,2,2-trifluoroethanol (TFE) are stretched at various draw ratios, locked-in the extended conformations, crosslinked by glutaraldehyde, and then mechanically analyzed in the longitudinal and transverse directions. Notably, the longitudinal modulus of the gelatin films that are cast from water or TFE linearly increases with draw ratio $\lambda$, accompanied by a substantial reduction in transverse modulus. When normalized by the Young's modulus of undrawn films, the transverse moduli as a function of draw ratio follow a power-law relationship $E / E_{0} \propto \lambda^{\alpha}$, where $\alpha \approx-2.5$ for the water-cast films and $\alpha \approx-1.5$ for the TFE-cast films. A Scanning Electron Microscopic analysis of the water- and TFE-cast films shows the formation of wrinkles and microcracks oriented in the drawing direction due to large deformations. Fourier Transform Infrared spectroscopy reveals the dominance of aggregated strands and $\beta$-sheets in the secondary structures of the gelatin films and the deformation-induced renaturation of triple-helical structures in the films. A model is thus proposed relate the observed mechanical anisotropy of drawn gelatin films to deformation-induced structural anisotropy.

Keywords: gelatin films, large deformation, mechanical anisotropy, longitudinal modulus, transverse modulus 


\section{Introduction}

Large deformations that are involved in the formation of many biomaterials have a significant impact on their structural and mechanical properties. For instance, polymer chains may be greatly extended and aligned during fiber spinning, as the solution experiences high shear [1] and/or longitudinal forces [2]. As the solvent is rapidly removed via evaporation and other mechanisms [3], the solution is solidified and the extension and alignment of polymer chains can be preserved to certain extent in the resulting fibers. Presumably due to the deformation-induced extension of polymer chains, formation of $\beta$-sheet crystals has been observed in wet-spun silk microfibers [4] and denaturation of collagen has been identified in electrospun collagen nanofibers [5].

The extension of polymer chains can affect the structural and mechanical properties of fibers in the longitudinal and transverse directions, leading to the formation of anisotropic fibers. Indeed, the tensile properties of wet- [6] and electrospun fibers [5] increased as the fiber diameter decreased. Such trends have been established as partially the result of an increased orientation of polymer chains in smaller fibers that experience larger deformation than their larger fibers. Previously, a nanoindentation study reveals that the indentation modulus of silkworm silk is about 19 times higher in the longitudinal direction than in the transverse direction [7]. The deformation-induced mechanical anisotropy may have important implications in the manufacturing and mechanical analysis of fiber-based biomaterials, but remains poorly understood.

Herein we analyze deformation-induced mechanical and structural anisotropy using gelatin films, which possesses a unique combination of features as a model system. First, gelatin films can be stretched to $200 \%$ strains in a water/ethanol mixture without failure. Second, the deformation-induced extension and alignments of gelatin chains can be locked-in and partially preserved by chemical crosslinking. Last, the large dimensions of gelatin films permit tensile analysis in the longitudinal and transverse directions. Previously, Bigi et al. reported that drawing partially restores the triple-helical structures [8] and 
improves the mechanical properties of gelatin films in the longitudinal direction [9]. Using the gelatin film model, we investigate deformation-induced mechanical anisotropy in the tensile mode, in a fashion similar to mechanical analysis of soft tissues [10] and tissue scaffolds [11]. Deformation-induced changes in microstructures and secondary structures that are responsible for the mechanical anisotropy of the drawn films are also examined.

\section{Materials and methods}

\subsection{Sample preparation}

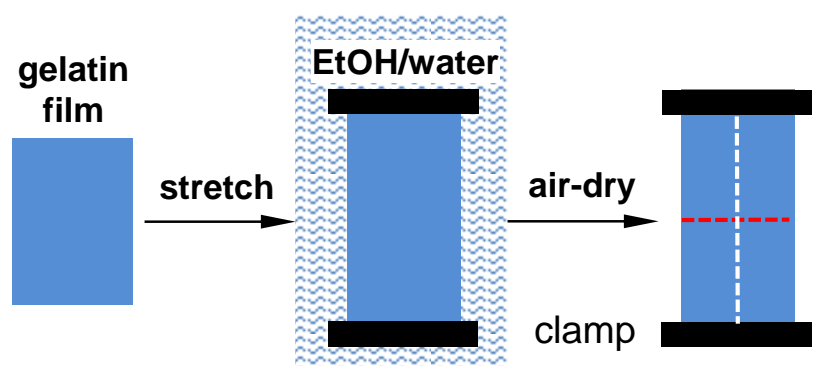

(a)

(b)

(c)

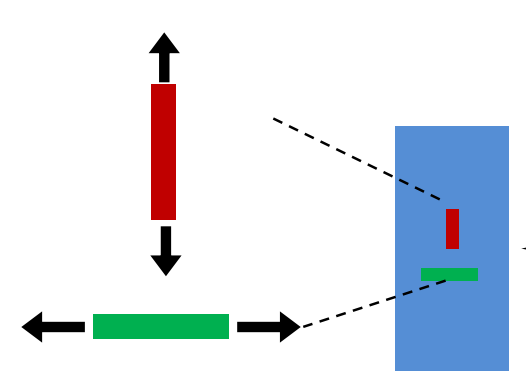

(e)

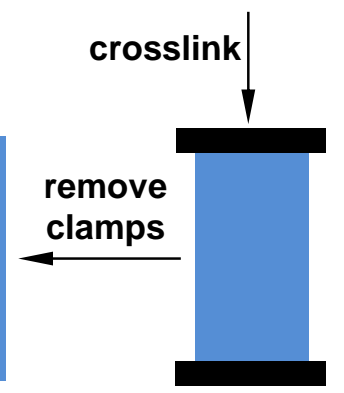

(d)

Figure 1. A schematic representation of sample preparation. (a) Casting gelatin films, (b) stretching the cast films in a mixture of ethanol and water, (c) air-drying the drawn films that remain to be clamped, (d) crosslinking the drawn and clamped films, and (e) preparing specimens from the longitudinal (marked in red) and transverse directions (marked in green) for uniaxial stress-strain analysis.

The process of sample preparation is illustrated in Fig. 1. Briefly, gelatin powder (Type A, approximate 300 Bloom, Sigma-Aldrich) was first dissolved at a concentration of $50 \mathrm{mg} / \mathrm{ml}$ in $31 \% \mathrm{v} / \mathrm{v} 2,2,2-$ trifluoroethanol (TFE) in water at room temperature, or in distilled, deionized water ( $d d$-water) at $60{ }^{\circ} \mathrm{C}$. 
The gelatin solution was mixed thoroughly by Vortex, and poured into polypropylene casting molds after air bubbles were removed by centrifugation. Solvent evaporation was performed in a chemical hood at room temperature for $24 \mathrm{~h}$ under ambient conditions. The cast gelatin films using the TFE/water mixture and water as solvents are called TFE-cast and water-cast films, respectively. Both the air-dried, TFE- and water-cast gelatin films were cut into rectangular strips with dimensions of $25 \mathrm{~mm} \times 40 \mathrm{~mm}$, which were immersed in a water/ethanol mixture at a 2:3 volume ratio, which provides a constant relative humidity of $75 \%$, for $24 \mathrm{~h}$. The immersed strips were elongated to various draw ratios (i.e., $\lambda=$ $1.5,2.0,2.5$, and 3.0) at a fixed rate of $10 \mathrm{~mm} / \mathrm{min}$ using a Test Resources 200R Pull Tester. Each drawn sample was air-dried for $24 \mathrm{~h}$ before being removed from the Pull Tester. Following a procedure reported previously [12], the samples were crosslinked with $10 \mathrm{ml}$ of glutaraldehyde (GTA, Mallinckrodt Baker) solution at a concentration of $2.5 \%(\mathrm{w} / \mathrm{v})$ in phosphate buffer at $\mathrm{pH} 7.4$ for $24 \mathrm{~h}$ at room temperature. To prevent the samples from shrinking in the GTA solution, each drawn sample was clamped to a Teflon block at its two ends. For a control study, non-stretched gelatin films were also crosslinked. The crosslinked samples, including undrawn and drawn films, were extensively rinsed with $d d$-water and air-dried for further use.

\subsection{Mechanical analysis}

Rectangular specimens of $2 \mathrm{~mm} \times 15 \mathrm{~mm}$ were cut from the GTA-crosslinked gelatin films. Five to six specimens could be cut from the central region of each drawn sample either in the longitudinal or transverse direction. Uniaxial tensile analysis of un- and drawn film specimens was performed using a PerkinElmer diamond DMA, and specimens were immersed in a jacketed beaker filled with 1X PBS at $37^{\circ} \mathrm{C}$. Displacement was applied at a fixed rate of $0.5 \mathrm{~mm} / \mathrm{min}$. Hydrated specimen thickness was typically $0.2 \mathrm{~mm}$, as measured using an optical microscope [13]. Specimens were monotonically stretched to failure so the deformability (i.e., strain at failure), ultimate tensile strength, and Young's modulus were obtained. 
The surface morphologies of un- and drawn gelatin films were examined using a Hitachi-S4800 field emission scanning electron microscope (FESEM). The specimens were sputter-coated with platinum for $45 \mathrm{sec}$ prior to SEM analysis with an accelerating voltage of $5 \mathrm{kV}$.

\subsection{Secondary structural analysis}

A Magna-IR 560 Nicolet spectrometer (Madison, WI) equipped with a CsI beam splitter and DTGSdetector was used to analyze the secondary structures of gelatin films. The spectrometer was purged continuously with dry air. FTIR spectra over the range of 4000-800 $\mathrm{cm}^{-1}$ were acquired using 400 scans of both sample and reference at a $4 \mathrm{~cm}^{-1}$ resolution. The FTIR data were collected on three replicate samples. Data collection was performed using Omnic processing software provided by Nicolet.

FTIR spectra of gelatin films in the spectral range of $1720-1580 \mathrm{~cm}^{-1}$ were smoothed with a 9-point smooth Savitzky-Golay function on GRAMS 8.0 and fitted with Gaussian band profiles. A baseline subtraction was also performed on GRAMS 8.0, and all the FTIR spectra were normalized by the areas of the amide I bands. Following a procedure reported in our previous study [14], the secondaryderivative and self-deconvolution methods were used to identify individual characteristic bands of the broadened amide I bands. Areas under individual bands normalized by the total area of the amide I band represent the percentage contents of secondary structures of gelatin films.

\section{Results and discussions}

\subsection{Drawn gelatin films display strong mechanical anisotropy}

As illustrated in Fig. 1, air-dried gelatin films were hydrated and stretched to various draw ratios (up to $\lambda=3$ ) in mixed water and ethanol at a 2:3 volume ratio. The large deformations are comparable to 
that experienced by silk microfibers in the wet-spinning process [4]. The deformation-induced extension and alignment of gelatin chains were preserved by crosslinking the stretched films using GTA. Specimens were cut from the drawn films in the longitudinal and transverse directions, and mechanically analyzed in the uniaxial tensile mode. Although a biaxial testing method may be more appropriate for anisotropic mechanical analysis [15-17], the uniaxial tensile approach provides a convenient way to evaluate the anisotropy of the drawn gelatin films using the currently available facilities. The drawn films displayed nearly linear responses to mechanical forces in both the longitudinal and transverse directions (Fig. 2). Accordingly, deformability (i.e., strain at failure) and tensile strength of the films in the longitudinal and transverse directions was evaluated from the stressstrain curves and expressed as mean \pm standard deviation, and the elastic modulus of the films was determined from the linear portion of the stress-strain curve and expressed as mean \pm standard deviation.

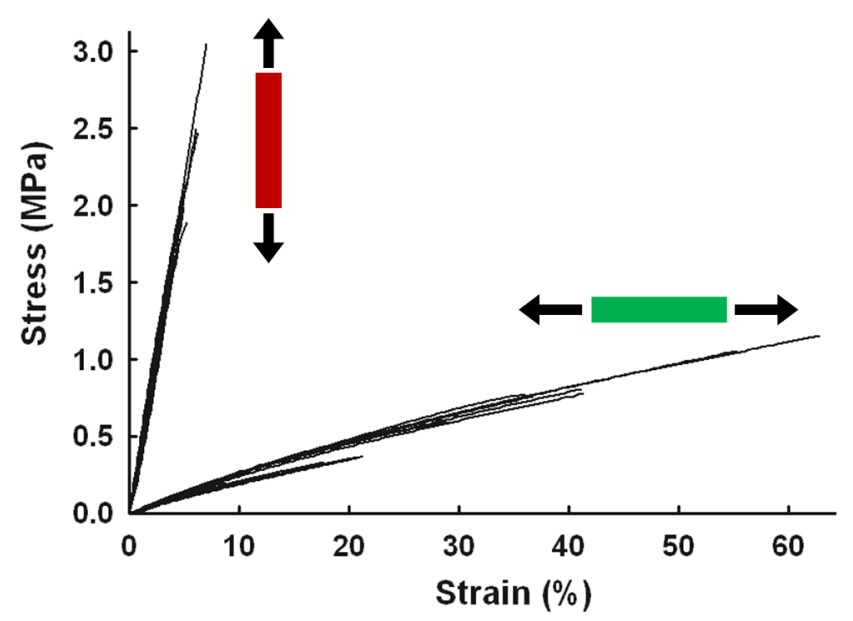

Figure 2. Uniaxial stress-strain analysis of TFE-cast gelatin films at a draw ratio $\lambda=3$.

As shown in Fig. 2, the deformation responses of drawn gelatin films are highly anisotropic. A uniaxial analysis of TFE-based films that were drawn to $\lambda=3$ prior to crosslinking reveals a longitudinal tensile modulus (denoted as $E \|)$ of $42.0 \pm 1.8 \mathrm{MPa}(\mathrm{n}=16, \mathrm{n}$ is the number of specimens tested), deformability of $4.57 \pm 1.21 \%$, and tensile strength of $1.84 \pm 0.52 \mathrm{MPa}$. In the transverse direction, the same film displayed a Young's modulus (denoted as $\left.E_{\perp}\right)$ of $1.93 \pm 0.22 \mathrm{MPa}(\mathrm{n}=11)$, deformability of $33.7 \pm$ 
$16.3 \%$, and tensile strength of $0.65 \pm 0.31 \mathrm{MPa}$. Significantly, the $E \|$ of the drawn gelatin films is 20 times higher than its transverse counterpart, $E_{\perp}$. On the other hand, the drawn films were more deformable in the transverse direction than in the longitudinal direction $(33.7 \pm 16.3 \%$ vs. $4.57 \pm$ $1.21 \%$ ). Moreover, the drawn films exhibited a large variation in the transverse deformability, varying from $17 \%$ to $62 \%$. Consequently, the transverse tensile strength was in the range of $0.33 \mathrm{MPa}$ to 1.15 MPa. Despite the large variations in transverse deformability and strength, the drawn films showed a small variation of about $10 \%$ in the transverse modulus.

\subsection{Draw ratio affects the longitudinal and transverse mechanical properties of gelatin films}
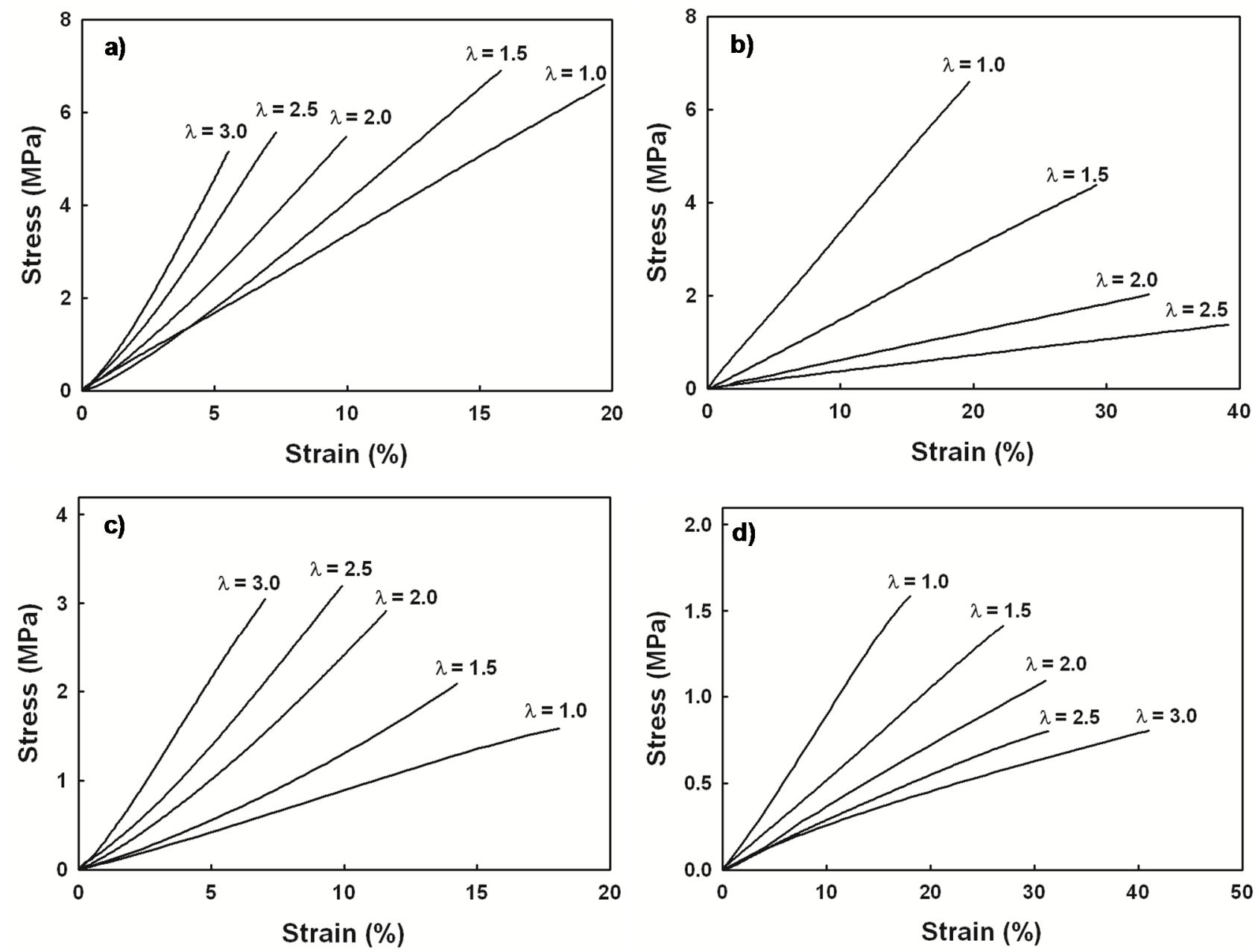

Figure 3. Representative stress-strain curves of gelatin films that were cast from different solvents, drawn at various ratios, and analyzed in the longitudinal and transverse directions. (a) Gelatin films were cast from water and analyzed in the longitudinal direction. (b) Gelatin films were cast from water 
and analyzed in the transverse direction. (c) Gelatin films were cast from TFE and analyzed in the longitudinal direction. (d) Gelatin films were cast from TFE and analyzed in the transverse direction.

The material responses of the drawn gelatin films to uniaxial tensile stress were analyzed in both the longitudinal and transverse directions (Fig. 3). At a draw ratio $\lambda=2$ or above, drawing reduced the longitudinal deformability of the water-cast gelatin films from about $20 \%$ to less than $10 \%$ (Fig. 3a). Accompanied with the reduction in longitudinal deformability, the $E \|$ of the water-cast films increased appreciably. As a result, the water-cast films drawn at different ratios showed comparable longitudinal strengths in the range of 5 to $7 \mathrm{MPa}$. In comparison to the non-drawn control, drawing nearly doubled the transverse deformability of the water-cast gelatin films at a drawing ratio $\lambda=2.5$, while both the transverse modulus and tensile strength of the water-cast films decreased considerably (Fig. 3b). When gelatin films were cast from TFE and drawn at various ratios, a reduction in longitudinal deformability and an increase in $E \|$ were also observed (Fig. 3c). Furthermore, the TFE-cast gelatin films displayed a noticeable increase in the longitudinal tensile strength when drawn at a ratio up to $\lambda=2$. Drawing also increased the transverse deformability of the TFE-cast films but decreased their $E_{\perp}$ and transverse tensile strength (Fig. 3d).

Evident by tensile stress-strain analysis, drawing increased $E \|$ and decreased the longitudinal deformability of gelatin films that were cast from either water or TFE; a reduction in $E_{\perp}$ and an increase in the transverse deformability were also observed upon drawing (Fig. 3a, 3c). Previously, Bigi et al. mechanically analyzed the longitudinal properties of the water-cast gelatin films in a 2:3 water/ethanol mixture and reported that drawing enhanced both the $E \|$ and longitudinal strength of the films but had little effect on their longitudinal deformability [9]. Here, we examined the mechanical properties of the water- and TFE-cast films in the longitudinal and transverse directions in physiologically relevant conditions (i.e., $37^{\circ} \mathrm{C}$ in $1 x$ PBS). Comparing our analysis with Bigi et al.'s study, it is clear that the material responses of the drawn gelatin films to external forces strongly depend on the testing 
conditions such as humidity. Specifically, the $E \|$ and longitudinal strength of the water-cast gelatin films obtained at $75 \%$ humidity were a few times higher than their counterparts obtained in PBS. However, the longitudinal deformability of the water-cast gelatin films was lower in a 2:3 water/ethanol mixture than in PBS. The observed humidity effects on deformability, strength and elastic modulus of the gelatin films were due to the hydration-induced plasticizing effects of polymer backbones. A similar hydration effect on elastic property was observed in micro- and nanoindentation analysis of mineralized collagen fibers [18]. We also reported that the full hydration of micrometer-diameter protein polymer fibers leads to a reduction in Young's modulus of 1,000 fold and an increase in deformation of several hundred times [6].

To further quantitatively analyze the deformation-induced mechanical anisotropy, the $E \|$ and $E_{\perp}$ of the water- and TFE-cast gelatin films as a function of draw ratio were determined. It is worthwhile mentioning that TFE [19] and hexafluoroisopropanol [20] are often used as polar solvents in the fabrication of gelatin nanofibers. Their ability to form strong hydrogen bonds with gelatin molecules, however, presents a challenge to the complete removal of these cytotoxic solvents from gelatin scaffolds. In addition, they are capable of breaking intra-/inter-molecular hydrogen bonds between gelatin molecules, potentially disrupting the triple-helical structures of gelatin that are derived from collagen. Previously, water and PBS have been explored as benign solvents for the fabrication of gelatin nanofibers [21]. In particular, gelatin nanofibrous scaffolds have been electrospun from aqueous solution either at elevated temperatures [22] or with the addition of salts and ethanol [21].

Here, the effects of deformation on the anisotropic mechanical properties of the water- and TFE-cast gelatin films are examined. As shown in Fig. 4a, the undrawn water-cast films possessed a Young's modulus of $33.1 \pm 1.7 \mathrm{MPa}$. As the films were drawn, their $E \|$ increased with draw ratio. Consequently, the $E \|$ of the films at $\lambda=3$ was three times higher than that of the undrawn films. Upon drawing, the water-cast films displayed a substantial reduction in $E_{\perp}$. For instance, the $E_{\perp}$ of the drawn films at $\lambda=$ 
2.5 was roughly one tenth of that of the undrawn films ( $3.4 \pm 0.2 \mathrm{MPa}$ vs. $33.1 \pm 1.7 \mathrm{MPa})$. Like the water-cast films, the TFE-cast films also exhibited a linear increase in $E \|$ and a significant reduction in $E_{\perp}$ upon drawing (Fig. 4b). Specifically, the $E \|$ of the TFE-cast films increased from $11.6 \pm 0.9 \mathrm{MPa}$ at $\lambda$ $=1$ to $42.0 \pm 1.8 \mathrm{MPa}$ at $\lambda=3$. In contrast, the $E_{\perp}$ of the TFE-cast films decreased from $11.6 \pm 0.9 \mathrm{MPa}$ at $\lambda=1$ to $1.9 \pm 0.2 \mathrm{MPa}$ at $\lambda=3$.
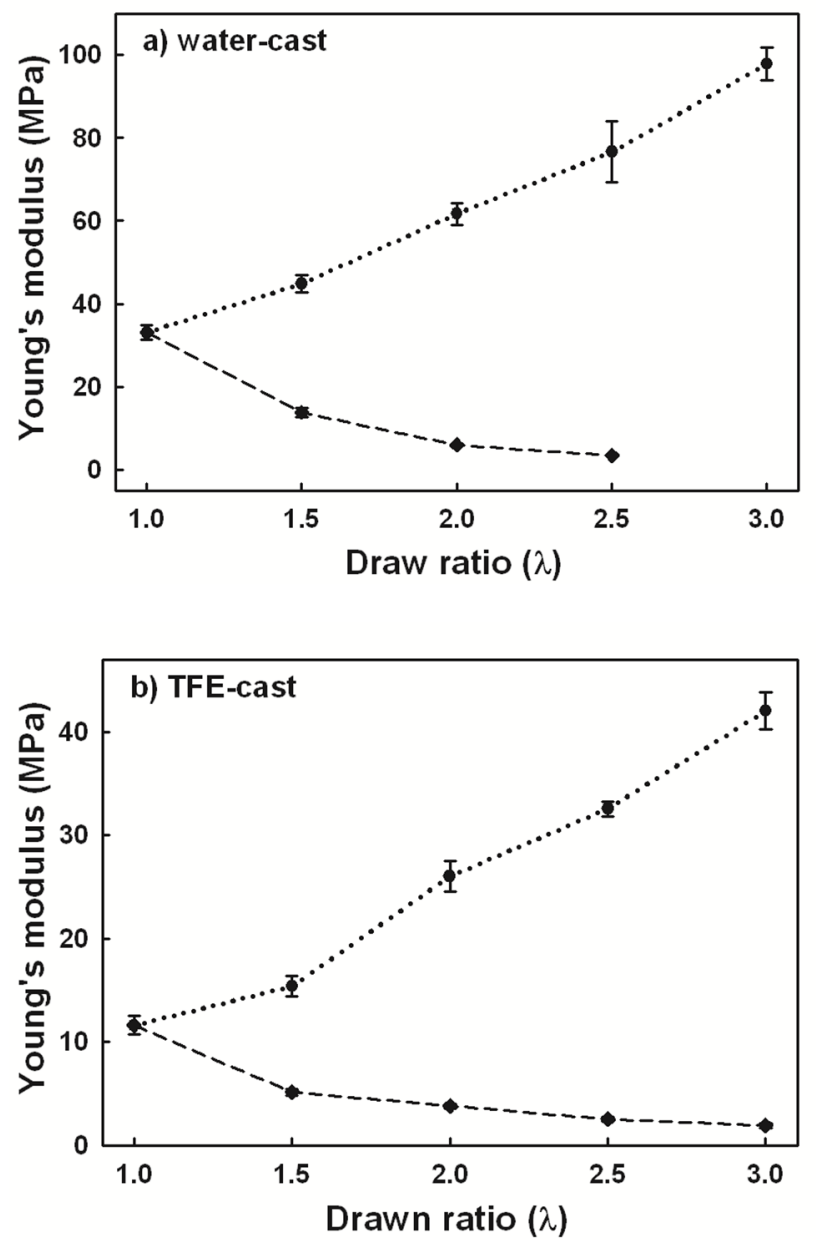

Fig. 4. The $E \|(\bullet)$ and $E_{\perp}(\bullet)$ of the water-cast (a) and TFE-cast gelatin films (b) as a function of draw ratio $\lambda$ ( $\lambda=1$ corresponds to the undrawn films).

Interestingly, the TFE-cast gelatin films possessed lower $E_{\|}$and $E_{\perp}$ than the water-cast films. While water preferentially solvates the hydrophilic blocks of protein polymers such as gelatin and favors micro-phase separation of the materials, TFE solvates both the hydrophilic and hydrophobic domains of protein polymers and promotes interpenetration of the domains. As a result, the TFE-cast elastinlike 
protein polymer (ELP) films displayed a higher Young's modulus and were more plastic than the watercast ELP films [23]. This is because both the hydrophilic and hydrophobic domains of ELP contribute to the deformation responses of the TFE-cast films. In the water-cast ELP films, however, only the soft, hydrophilic domains are deformed while the stiff, hydrophobic blocks function as physical cross-linkers. Here, the improved mechanical properties of the water-cast gelatin films over the TFE-cast films may be due to a better preservation of the secondary and/or higher-ordered structures of protein polymers when water is used as a solvent. Previously, Termonia reported that protein crystals contribute greatly to the overall strength of spider silk [24]. Likely, protein aggregates/crystals also considerably affect the mechanical properties of the gelatin films. However, TFE as a strong polar solvent may disrupt the higher-ordered structures of gelatin and thus compromise the mechanical properties of the TFE-cast films.

\subsection{A power-law relationship was observed between normalized Young's modulus of gelatin}

\section{films and draw ratio}

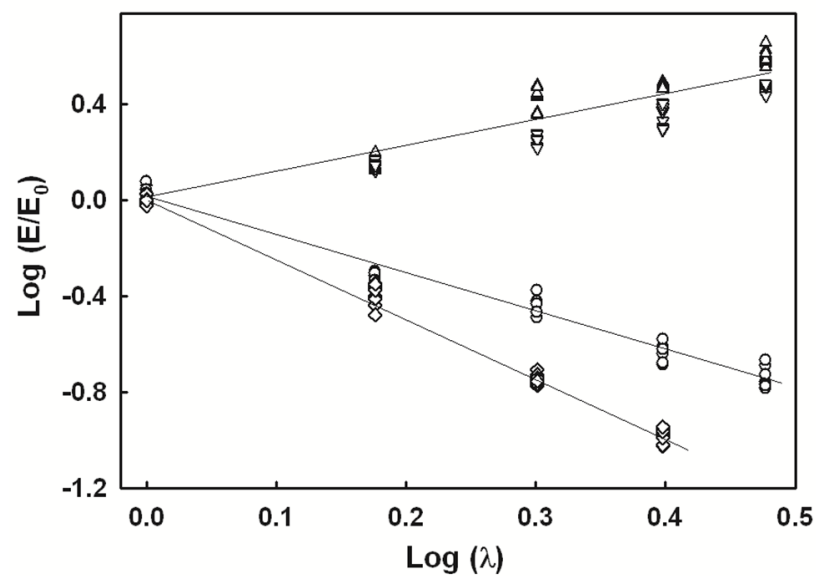

Figure 5. Normalized elastic modulus as a function of draw ratio. The $E \|(\Delta)$ and $E_{\perp}(\diamond)$ of the watercast gelatin films were normalized by the Young's modulus of the undrawn water-cast films. The $E \|(\nabla)$ and $E_{\perp}(O)$ of the TFE-cast gelatin films were normalized by the Young's modulus of the undrawn TFE-cast films. 
To gain further insights into deformation-induced mechanical anisotropy, the $E \|$ and $E_{\perp}$ of the waterand TFE-cast gelatin films were normalized using the Young's modulus of the respective undrawn films (denoted as $E_{0}$ ), leading to $\hat{E}=E / E_{0}$, in which $\hat{E}$ is the normalized $E \|$ or $E_{\perp}$. The normalized moduli were plotted against draw ratio in the double-logarithmic scales (Fig. 5). Strikingly, linear relationships between $\log (\hat{E})$ and $\log (\lambda)$ were observed in all four cases:

$$
\log (\hat{E})=\alpha \cdot \log (\lambda)
$$

where $\alpha$ is the slope of the linear double-logarithmic curve. For the four cases, $\alpha$ is listed in Table 1.

Table 1. Slopes $\alpha$ of the linear double-logarithmic curves plotted in Fig. 5.

\begin{tabular}{ccccc}
\hline solvent/direction & water/longitudinal & TFE/longitudinal & water/transverse & TFE/transverse \\
$\alpha$ & 1.07 & 1.07 & -2.5 & -1.5 \\
\hline
\end{tabular}

Specifically, $\log (\hat{E})=1.07 \times \log (\lambda)$ was established for the $E \|$ of the water- and TFE-cast gelatin films, $\log (\hat{E})=-2.5 \times \log (\lambda)$ was identified for the $E_{\perp}$ of the water-cast films, and $\log (\widehat{E})=-1.5 \times \log (\lambda)$ was established for the $E_{\perp}$ of the TFE-cast films. Accordingly, the normalized modulus $\hat{E}$ as a function of draw ratio follows a simple power law:

$$
\hat{E}=\lambda^{\alpha}
$$

where $\alpha \approx 1.0$ for the $E \|$ of the water- and TFE-cast gelatin films, $\alpha=-2.5$ for the $E_{\perp}$ of the water-cast films, and $\alpha=-1.5$ for the $E_{\perp}$ of the TFE-cast films. The scaling analysis unambiguously confirms a linear increase in $E \|$ and a substantial reduction in $E_{\perp}$ in the water- and TFE-cast gelatin films. Interestingly, the water-cast gelatin films displayed a more rapid decrease in $E_{\perp}$ than the TFE-cast films.

\subsection{Deformation induces structural anisotropy of gelatin films}




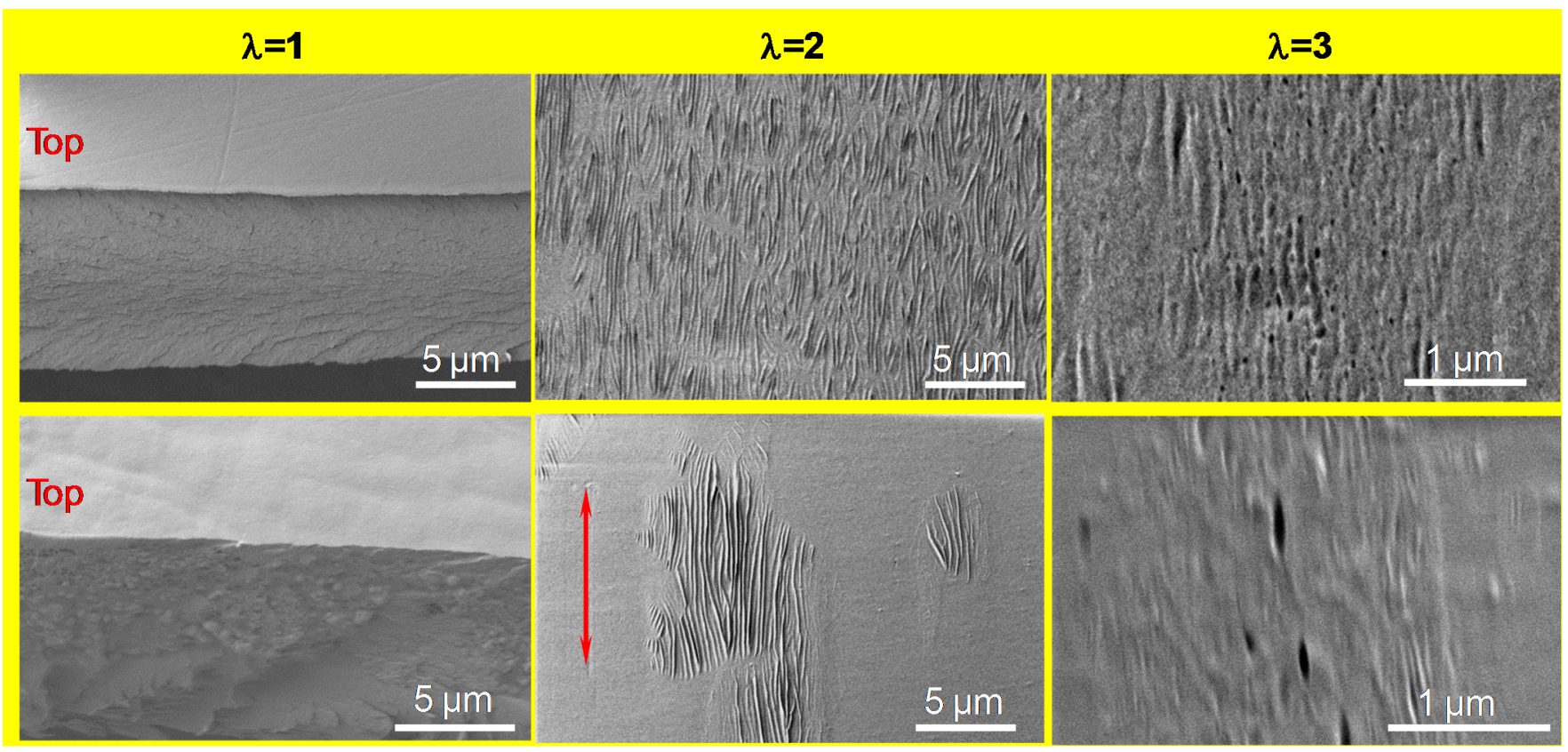

Figure 6. SEM images of water- (top panel) and TFE-cast gelatin films (bottom panel) at various draw ratios. Both the top and cross sectional surfaces of the undrawn films were examined (left panel, $\lambda=1$ ), while the top surfaces of the drawn films were analyzed (central panel, $\lambda=2$; right panel $\lambda=3$ ). The red arrow indicates the direction of deformation.

To identify underlying mechanisms for the drawing-induced mechanical anisotropy, the surface morphologies and microstructures of the water- and TFE-cast films were analyzed using SEM. As shown in Figure 6, the undrawn water- and TFE-cast gelatin films displayed smooth surfaces. Upon drawing, wrinkles appeared on the surfaces of the water-cast films in the drawing direction. Wrinkles were observed in some areas of the surfaces of the TFE-cast films. At a draw ratio of $\lambda=2$, most wrinkles appearing on the water- and TFE-cast films were several micrometers in length. The drawing-induced formation of wrinkles in gelatin films can be well described by a packingconstraint theory of wrinkling [25]. According to the wrinkling theory, thin skins can form on the surfaces of a gelatin film during the drying process, and the resulting skins are much stiffer than the bulk material, which forms the substrate. Under tensile deformation in the drawing process, the incompatible mechanical properties of the skin and substrate prevent a uniform lateral contraction of the gelatin films, leading to the accumulation of small compressive stresses in the skin in the lateral 
direction; once the tensile deformation exceeds a critical value, the accumulated compressive stress will cause the gelatin skin to buckle into a series of periodic wrinkles in the lateral direction [26]. The wrinkle formation is highly sensitive to the dehydration process. Specifically, a slow drying process facilitates the formation of wrinkles in gelatin films. Indeed, few wrinkles were observed in the TFEcast gelatin films, compared to the water-cast films (Figure 5). This suggests that the fast evaporation of TFE minimized the generation of skins during the film casting process and the subsequent formation of wrinkles upon drawing.

The SEM analysis clearly shows that large deformations (e.g., $\lambda=3$ ) induce micrometer-sized damages to the gelatin films, such as microcracks/elongated microvoids in water-cast films and microcracks in TFE-cast films (Figure 6). Further, the microcracks/elongated microvoids were formed and/or aligned along the drawing direction. Such a spatial distribution compromises material integrity and impacts the failure of gelatin films more greatly in the transverse direction than in the longitudinal direction. When the drawn films are stretched in the transverse direction, the microcracks/elongated microvoids would grow and coalesce in the drawing direction, leading the failure of the films. It is well known that the ultimate tensile strength of a material can be drastically reduced by the extension and coalescence of microcracks [27], while Young's modulus is less sensitive to the density and spatial distribution of microcracks.
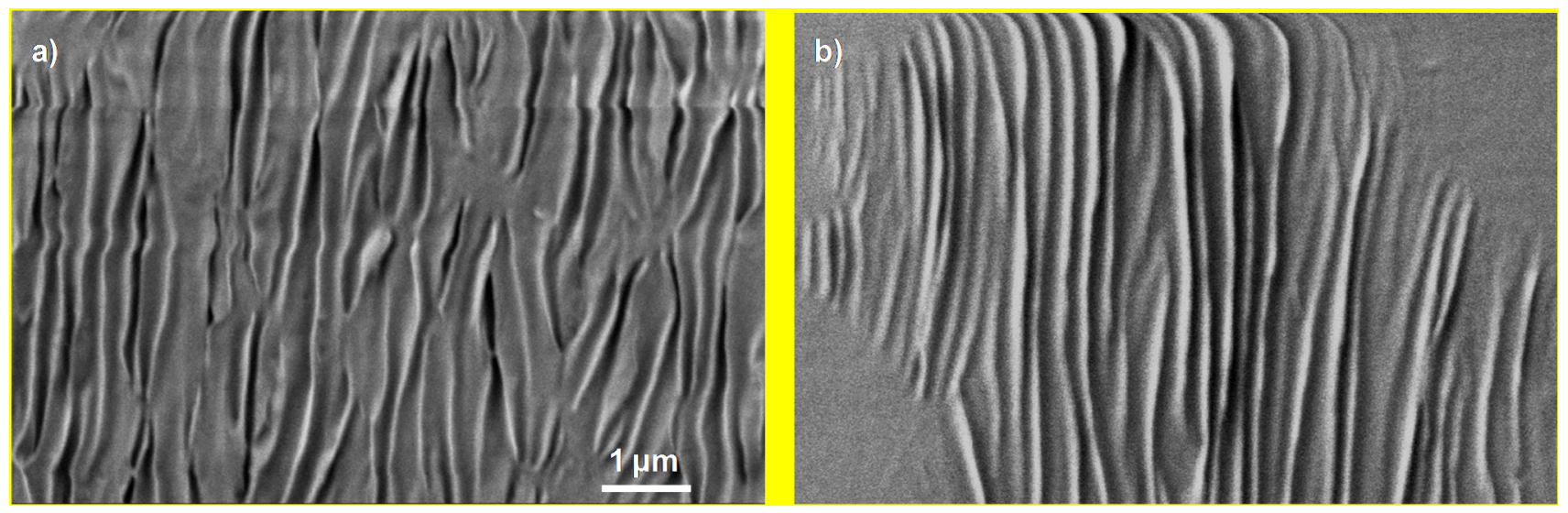

Figure 7. A close SEM examination of wrinkles formed on the water- (a) and TFE-cast (b) gelatin films prepared at a draw ratio of $\lambda=2.0$. 
A close examination of the SEM images reveals that distance between the wrinkles, namely the wavelength of wrinkles, was $200 \sim 400 \mathrm{~nm}$ for the water-cast films (Figure 7a) and $200 \sim 300 \mathrm{~nm}$ for the TFE-cast films (Figure $7 b$ ). The wrinkle wavelength $l$ can be related to the thickness $(h)$ of wrinkles in the gelatin films as $l \sim h\left(E_{\mathrm{s}} / E_{\mathrm{b}}\right)^{1 / 3}$, where $E_{\mathrm{s}}$ and $E_{\mathrm{b}}$ are the Young's moduli of the gelatin skin and bulk material, respectively [26]. If it is assumed that $E_{\mathrm{s}} \sim 10 E_{\mathrm{b}}$, the wrinkling theory then suggests the formation of skins of $100 \sim 200 \mathrm{~nm}$ thickness in the water-cast gelatin films and skins of $100 \sim 150 \mathrm{~nm}$ thickness in the TFE-cast films. When gelatin films were prepared at a draw ratio of $\lambda=3$, a reduction in the density and height of wrinkles was observed on the surface of the films, compared to specimens prepared at a draw ratio of $\lambda=2$. A plausible explanation is that deformation-induced micro-damages in the films reduced the accumulated residual stress, which was responsible for the formation of wrinkles.

\subsection{Deformation partially restores triple-helical structures in the drawn gelatin films}

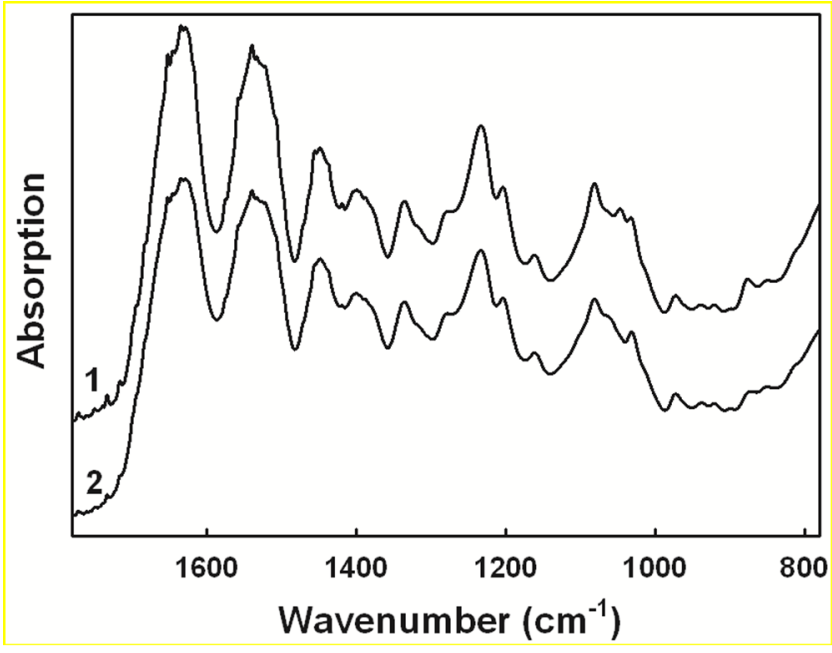

Figure 8. FTIR spectra of water- (1) and TFE-cast (2) gelatin films in the spectral range of 1800-800 $\mathrm{cm}^{-1}$. 


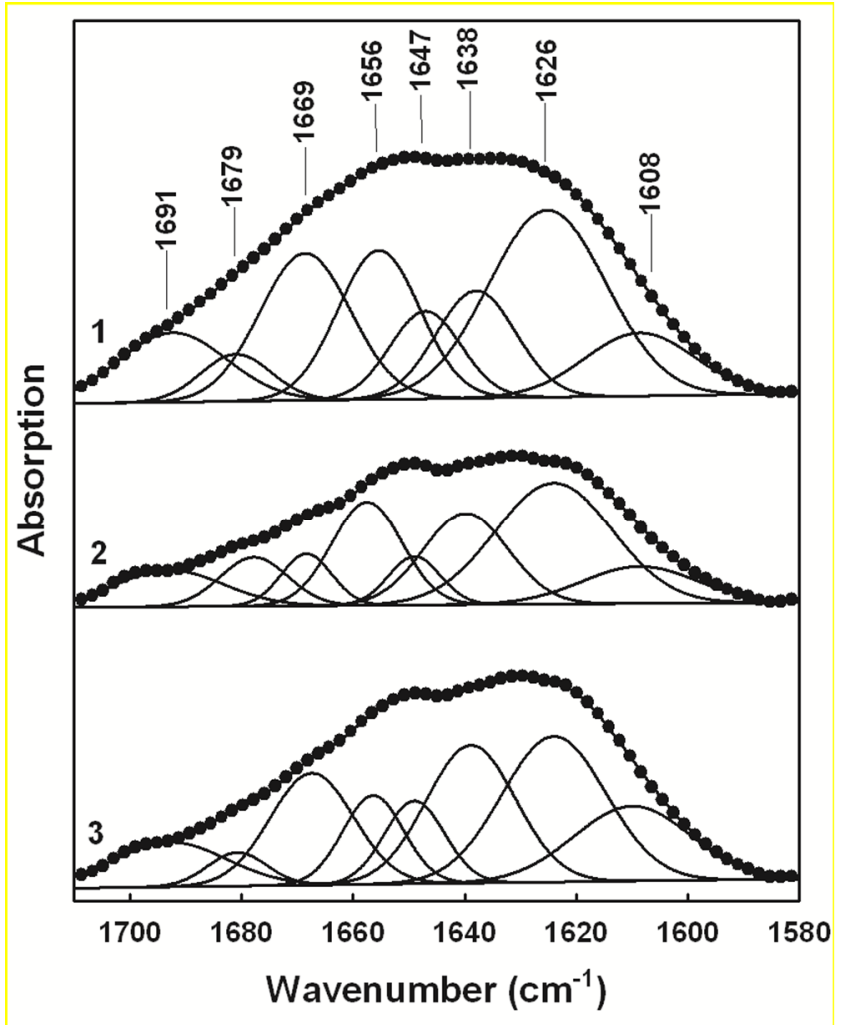

Figure 9. FTIR amide I spectra of the TFE-cast gelatin films at draw ratios of $\lambda=1,2$, and 3 were fitted with Gaussian band profiles.

Because of its collagenous origin, gelatin may possess triple-helical structures. Evident by X-ray and differential scanning calorimetry analyses, stretching a gelatin film can lead to a partial renaturation of its triple helices, which are lost in physical and chemical process of collagenous tissues [8, 9]. Herein, FTIR was employed to investigate both the solvent and drawing effects on the secondary structures of gelatin films. Specifically, an absorption at $1635 \mathrm{~cm}^{-1}$ and a shoulder at $1655 \mathrm{~cm}^{-1}$ are attributed to amide I (CO stretching) and absorptions at 1540 and $1231 \mathrm{~cm}^{-1}$ are ascribed to amide II (CN stretching and NH bending) and amide III, respectively. Bands at 1448, 1401, 1336, 1278, and $1203 \mathrm{~cm}^{-1}$ are attributed to $\mathrm{CH}_{2}$ and $\mathrm{CH}_{3}$ wagging, scissoring, bending, and stretching $[6,14,28]$. Interestingly, the water- and TFE-cast gelatin films exhibited nearly identical FTIR spectra (Figure 8).

Following a previously described procedure, quantitative structural information on gelatin films was derived by curve fitting of the FTIR amide I bands with Gaussian band profiles [14]. As pointed out by Goormaghtigh et el., special attention needs to be paid to an FTIR-based secondary structural analysis 
of proteins that are rich in helical structures, including $\alpha$ - and triple helices [29]. Petibois et al. have proposed the use of eight Gaussian bands at 1691, 1679, 1669, 1656, 1647, 1638, 1626, and $1608 \mathrm{~cm}^{-1}$ for the quantitative FTIR analysis of collagenous tissues [28]. Specifically, the bands at 1638, 1647, and $1656 \mathrm{~cm}^{-1}$ were assigned to triple helix [28], unordered structure $[14,28]$ and $\alpha$-helix $[28,30]$, respectively. Bands at $1610,1626,1679$, and $1691 \mathrm{~cm}^{-1}$ were assigned to $\beta$-sheets $[14,28,30]$ and $\beta$ strand aggregates $[14,31]$, while bands at $1669 \mathrm{~cm}^{-1}$ were assigned to $\beta$-turns. Using the eight Gaussian bands, the FTIR amide I spectra of the TFE-cast films at $\lambda=1,2$, and 3 were curve-fitted and areas under the Gaussian bands were used to evaluate the percentages of secondary structures (Figure 9). Table 2. Percentages of secondary structures as determined by curve-fitting of the amide I bands of the TFE-cast gelatin films

\begin{tabular}{ccccc}
\hline draw ratio & triple-helix/ $\alpha$-helix & $\beta$-sheets \& aggregates & $\beta$-turns & unordered structure \\
$\mathbf{1}$ & $10.7 / 15.3$ & 49.5 & 16.7 & 7.8 \\
$\mathbf{2}$ & $17.0 / 15.9$ & 55.8 & 5.7 & 5.5 \\
$\mathbf{3}$ & $19.6 / 8.6$ & 48.9 & 14.9 & 7.9 \\
\hline
\end{tabular}

As summarized in Table 2, the dominating secondary structures of the non-drawn gelatin films include $\beta$-sheets/aggregated strands (49.5\%) and helical structures $(10.7 \%$ triple helix and $15.3 \% \alpha-$ helix). Upon drawing at $100 \%$ strains, $\beta$-turns and the unordered structure were partially converted into triple helices and $\beta$-sheets/aggregated strands. As a result, the content of triple-helical structures was increased from $10.7 \%$ to $17.0 \%$. Further stretching the gelatin films slightly disrupted $\alpha$-helices and $\beta$ sheet structures, leading to a moderate increase in $\beta$-turns and the unordered structure. However, a slight gain in triple-helical structures was still observed. Consistent with Bigi et al.'s differential scanning calorimetry and X-ray analyses [8,9], the FTIR spectroscopy confirms the drawing-induced renaturation of triple-helical structures in the gelatin films. Additionally, this study also reveals the dominance of extended $\beta$-sheets and strongly hydrogen-bonded aggregates in gelatin films, which is due to the denaturation of collagenous tissues in gelatin production. 


\subsection{A model is proposed for the deformation-induced mechanical anisotropy of gelatin films}

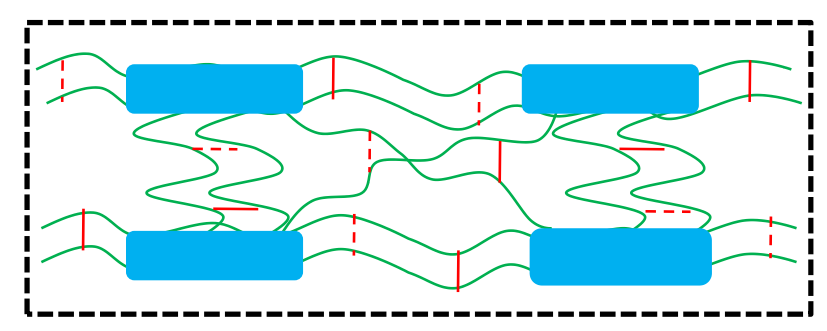

Figure 10. Deformation-induced structural anisotropy. At the molecular scale, the flexible chains (in green) linking protein aggregates/crystals (in light blue) are extended in the longitudinal direction but cramped in the transverse direction. The extended and cramped chains are locked in their conformations via the breakage and reformation of hydrogen bonds (red dashed lines) as well as the formation of chemical bonds (red solid lines).

The polymer chains of gelatin films may be grouped into two categories: molecularly flexible chains and polymer chains that form protein aggregates/crystals. The flexible chains include $\beta$-turn, helical, and unordered structures. The protein aggregates/crystals function as physical cross-links and reinforce the film in a fashion similar to carbon black in elastomers [24]. Conversion exists between flexible chains and protein aggregates: flexible chains can be aligned and crystallized or aggregated into protein crystals/aggregates and the disruption of intra/intermolecular hydrogen bonds may break protein aggregates/crystals into flexible chains. Prior to drawing a gelatin film, the flexible chains and protein aggregates/crystals are randomly orientated. Upon drawing, the flexible chains and protein aggregates will be deformed and aligned. In particular, the flexible chains that link the protein aggregates in the longitudinal direction may be highly extended to transmit forces, while the chains in the lateral direction may be cramped (Fig. 10). Both the strained and cramped chains should recoil, recovering their original conformations, when the external load is removed. As the film is held at a constant draw ratio for $24 \mathrm{~h}$ and then crosslinked using GTA, however, the entanglements and conformations of the extended and cramped chains will be rearranged to relax the internal stress. This is achieved through the breakage and 
reformation of hydrogen bonds between gelatin molecules as well as the formation of chemical bonds due to GTA crosslinking. As a consequence, an anisotropic network of flexible chains and protein aggregates will be formed (Fig. 10). Since the flexible chains are already aligned and extended in the longitudinal direction, the film will be more resistant to longitudinal stretching. In contrast, transverse stretching of the film can be readily accommodated by extension of the cramped chains that link the protein aggregates/crystals in the transverse direction. It is thus anticipated that a drawn film will display a higher Young's modulus and lower deformability in the drawing direction, but a lower Young's modulus and higher deformability in the transverse direction.

\section{Conclusions}

Deformation-induced anisotropy has been mechanically and structurally analyzed using a gelatin film model. The longitudinal tensile modulus of the gelatin films linearly increases with draw ratio but the transverse modulus greatly decreases, following a power law $E \propto \lambda^{\alpha}$, where $\alpha \approx-2.5$ for the water-cast films and $\alpha \approx-1.5$ for the TFE-cast films. Deformability is increased in the transverse direction but decreased in the longitudinal direction. SEM analyses of the drawn gelatin films show the generation of wrinkles and microcracks during the drawing process, and the alignment of wrinkles and microcracks in the drawing direction. FTIR spectroscopy identifies aggregated strands and $\beta$-sheets as the dominated secondary structures of the gelatin films and a partial renaturation of triple-helical structures owing to drawing. Taken together, drawing likely induces the formation of an anisotropic network of protein aggregates/crystals and flexible chains at the molecular scale as well as anisotropic microstructures at the microscale. Specifically, flexible polymer chains are extended and aligned in the longitudinal direction, increasing longitudinal modulus, but cramped in the transverse direction, reducing transverse modulus and increasing transverse deformability.

\section{Acknowledgment}


This work was supported by the National Institute of Health (EB009160) and National Science

Foundation (CMMI0855890).

\section{References}

[1] Jin H. J., Kaplan D. L.: Mechanism of silk processing in insects and spiders. Nature, 424, $1057-$ 1061 (2003).

[2] Han T., Yarin A. L., Reneker D. H.: Viscoelastic electrospun jets: Initial stresses and elongational rheometry. Polymer, 49, 1651-1658 (2008).

[3] Vollrath F., Knight D. P.: Liquid crystalline spinning of spider silk. Nature, 410, 541-548 (2001).

[4] Liivak O., Blye A., Shah N., Jelinski L. W.: A microfabricated wet-spinning apparatus to spin fibers of silk proteins. Structure-property correlations. Macromolecules, 31, 2947-2951 (1998).

[5] Yang L., Fitie C. F. C., van der Werf K. O., Bennink M. L., Dijkstra P. J., Feijen J.: Mechanical properties of single electrospun collagen type I fibers. Biomaterials, 29, 955-962 (2008).

[6] Qiu W. G., Teng W. B., Cappello J., Wu X. Y.: Wet-Spinning of Recombinant Silk-Elastin-Like Protein Polymer Fibers with High Tensile Strength and High Deformability. Biomacromolecules, 10, 602-608 (2009).

[7] Zhang K., Si F. W., Duan H. L., Wang J.: Microstructures and mechanical properties of silks of silkworm and honeybee. Acta Biomaterialia, 6, 2165-2171 (2010).

DOI: DOI 10.1016/j.actbio.2009.12.030.

[8] Bigi A., Borghi M., Cojazzi G., Fichera A. M., Panzavolta S., Roveri N.: Structural and mechanical properties of crosslinked drawn gelatin films. Journal of Thermal Analysis and Calorimetry, 61, 451-459 (2000).

[9] Bigi A., Bracci B., Cojazzi G., Panzavolta S., Roveri N.: Drawn gelatin films with improved mechanical properties. Biomaterials, 19, 2335-2340 (1998).

[10] Zhu J. T., Wang X. Z., He C. C., Wang H. L.: Mechanical properties, anisotropic swelling behaviours and structures of jellyfish mesogloea. Journal of the Mechanical Behavior of Biomedical Materials, 6, 63-73 (2012).

DOI: 10.1016/j.jmbbm.2011.10.005.

[11] Caliari S. R., Weisgerber D. W., Ramirez M. A., Kelkhoff D. O., Harley B. A. C.: The influence of collagen-glycosaminoglycan scaffold relative density and microstructural anisotropy on tenocyte bioactivity and transcriptomic stability. Journal of the Mechanical Behavior of Biomedical Materials, 11, 27-40 (2012).

[12] Teng W. B., Cappello J., Wu X. Y.: Recombinant Silk-Elastinlike Protein Polymer Displays Elasticity Comparable to Elastin. Biomacromolecules, 10, 3028-3036 (2009). 
[13] Qiu W. G., Huang Y. D., Teng W. B., Cohn C. M., Cappello J., Wu X. Y.: Complete Recombinant Silk-Elastinlike Protein-Based Tissue Scaffold. Biomacromolecules, 11, 3219-3227 (2010).

DOI: $10.1021 / \mathrm{bm} 100469 \mathrm{w}$.

[14] Teng W. B., Huang Y. D., Cappello J., Wu X. Y.: Optically transparent recombinant silkelastinlike protein polymer films. Journal of Physical Chemistry B, 115, 1608-1615 (2011).

[15] Sacks M. S., Sun W.: Multiaxial mechanical behavior of biological materials. Annual Review of Biomedical Engineering, 5, 251-284 (2003).

[16] Zou Y., Zhang Y. H.: An Experimental and Theoretical Study on the Anisotropy of Elastin Network. Annals of Biomedical Engineering, 37, 1572-1583 (2009).

DOI: DOI 10.1007/s10439-009-9724-z.

[17] Pham T., Sun W.: Comparison of biaxial mechanical properties of coronary sinus tissues from porcine, ovine and aged human species. Journal of the Mechanical Behavior of Biomedical Materials, 6, 21-29 (2012).

DOI: 10.1016/j.jmbbm.2011.09.001.

[18] Spiesz E. M., Roschger P., Zysset P. K.: Elastic anisotropy of uniaxial mineralized collagen fibers measured using two-directional indentation. Effects of hydration state and indentation depth. Journal of the Mechanical Behavior of Biomedical Materials, 12, 20-28 (2012).

[19] Huang Z. M., Zhang Y. Z., Ramakrishna S., Lim C. T.: Electrospinning and mechanical characterization of gelatin nanofibers. Polymer, 45, 5361-5368 (2004).

[20] Kim H. W., Song J. H., Kim H. E.: Nanoriber generation of gelatin-hydroxyapatite biomimetics for guided tissue regeneration. Advanced Functional Materials, 15, 1988-1994 (2005).

DOI: $10.1002 /$ adfm.200500116.

[21] Zha Z. B., Teng W. B., Markle V., Dai Z. F., Wu X. Y.: Fabrication of gelatin nanofibrous scaffolds using ethanol/phosphate buffer saline as a benign solvent. Biopolymers, 97, 1026-1036 (2012).

DOI: $10.1002 /$ bip.22120.

[22] Zhang S., Huang Y. Q., Yang X. P., Mei F., Ma Q., Chen G. Q., Ryu S., Deng X. L.: Gelatin nanofibrous membrane fabricated by electrospinning of aqueous gelatin solution for guided tissue regeneration. Journal of Biomedical Materials Research Part A, 90A, 671-679 (2009).

[23] Wu X. Y., Sallach R., Haller C. A., Caves J. A., Nagapudi K., Conticello V. P., Levenston M. E., Chaikof E. L.: Alterations in physical cross-linking modulate mechanical properties of two-phase protein polymer networks. Biomacromolecules, 6, 3037-3044 (2005).

[24] Termonia Y.: Molecular Modeling of Spider Silk Elasticity. Macromolecules, 27, 7378-7381 (1994).

[25] Cerda E., Mahadevan L.: Geometry and physics of wrinkling. Physical Review Letters, 90(2003). 
DOI: Artn 074302

Doi 10.1103/Physrevlett.90.074302.

[26] Rizzieri R., Mahadevan L., Vaziri A., Donald A.: Superficial wrinkles in stretched, drying gelatin films. Langmuir, 22, 3622-3626 (2006).

DOI: Doi 10.1021/La052343m.

[27] Lawn B. R., Fracture of brittle solids. 2nd ed. Cambridge solid state science series. 1993, Cambridge ; New York: Cambridge University Press. xix, 378 p.

[28] Petibois C., Gouspillou G., Wehbe K., Delage J. P., Deleris G.: Analysis of type I and IV collagens by FT-IR spectroscopy and imaging for a molecular investigation of skeletal muscle connective tissue. Analytical and Bioanalytical Chemistry, 386, 1961-1966 (2006).

DOI: DOI 10.1007/s00216-006-0828-0.

[29] Goormaghtigh E., Ruysschaert J. M., Raussens V.: Evaluation of the information content in infrared spectra for protein secondary structure determination. Biophysical Journal, 90, 2946-2957 (2006).

DOI: DOI 10.1529/biophysj.105.072017.

[30] Taddei P., Monti P.: Vibrational infrared conformational studies of model peptides representing the semicrystalline domains of Bombyx mori silk fibroin. Biopolymers, 78, 249-258 (2005).

[31] Ami D., Natalello A., Taylor G., Tonon G., Doglia S. M.: Structural analysis of protein inclusion bodies by Fourier transform infrared microspectroscopy. Biochimica Et Biophysica Acta-Proteins and Proteomics, 1764, 793-799 (2006).

DOI: DOI 10.1016/j.bbapap.2005.12.005. 